\title{
Experimental realization of hyper-entangled two-photon states
}

\author{
C. Cinelli, M. Barbieri, F. De Martini and P. Mataloni \\ Dipartimento di Fisica and \\ Istituto Nazionale per la Fisica della Materia, \\ Università di Roma La Sapienza, \\ Roma, 00185 - Italy \\ e-mail: paolo.mataloni@uniroma1.it
}

\begin{abstract}
We report on the the experimental realization of hyper-entangled two photon states, entangled in polarization and momentum. These states are produced by a high brilliance parametric source of entangled photon pairs with peculiar characteristics of flexibility in terms of state generation. The quality of the entanglement in the two degrees of freedom has been tested by multimode Hong-Ou-Mandel interferometry.
\end{abstract}

\section{Introduction}

The modern science of quantum information (QI) squarely lies on the concept and on the applications of entanglement which represents the basic process underlying quantum computation [1, quantum teleportation [2] and some quantum cryptographic schemes [3]. The most successful and reliable applications of entanglement, such as, for instance, quantum dense coding [4], teleportation [5], entanglement swapping [6], have been obtained so far in quantum optics, by exploiting the process of Spontaneous Parametric Down Conversion (SPDC) [7]. Here a pair of photons, at wavelengths (wl) $\lambda_{1}$ and $\lambda_{2}$ and momenta $\hbar \mathbf{k}_{1}$ and $\hbar \mathbf{k}_{2}$, may be generated by a nonlinear (NL) optical 
crystal shined by a pump laser beam at wl $\lambda_{p}$. The conservation of energy, $\lambda_{1}^{-1}+\lambda_{2}^{-1}=\lambda_{p}^{-1}$, and of the momentum, $\mathbf{k}_{1}+\mathbf{k}_{2}=\mathbf{k}_{p}$, leads to frequency and $\mathbf{k}$-vector correlation of the emitted photons. The electromagnetic modes are associated with qubits which are generally encoded by the field polarization. In this framework the bi-partite $2 \times 2$ Hilbert space is spanned by the four maximally entangled Bell-state basis,

$$
\begin{aligned}
& \left|\Psi^{ \pm}\right\rangle=\frac{1}{\sqrt{2}}\left(\left|H_{1}, V_{2}\right\rangle \pm\left|V_{1}, H_{2}\right\rangle\right), \\
& \left|\Phi^{ \pm}\right\rangle=\frac{1}{\sqrt{2}}\left(\left|H_{1}, H_{2}\right\rangle \pm\left|V_{1}, V_{2}\right\rangle\right),
\end{aligned}
$$

expressed in the horizontal $(H)$ and vertical $(V)$ polarization basis.

It is worth noting that the above referred QI schemes [2, 4, 5, 6] can be completely realized only if the entire set of Bell state measurements is performed by distinguishing among the four orthogonal Bell states with 100\% efficiency. Unfortunately enough, this is impossible with standard linear optics, and the maximum attainable value of efficiency is $50 \%$ [

Entangled states, prepared in more than one degree of freedom, or hyperentangled states, have been proposed for optical Bell state analysis, in particular by entangling two photons in polarization and momentum 9]. Different schemes have been proposed which are based, as SPDC source, either on type II non collinear phase matching [10, 11, or on the type I two crystal system [12, 13]. However, the experimental realization of this kind of hyper-entangled states has never been realized so far.

In the present work we report on the realization of the simultaneous polarization and momentum entanglement of photon pairs generated by a high brilliance SPDC source, recently developed by us [14, 15], with peculiar characteristics of flexibility in terms of state generation.

\section{Experimental set-up}

The high brilliance source of entanglement is sketched in Fig. 1 [14, 15. A Type I, .5mm thick, $\beta$-barium-borate (BBO) crystal is excited in two opposite directions by a back-reflected $V$-polarized mode-locked Ti-Sapphire femtosecond laser $(P=400 \mathrm{~mW}$, rep. rate $=76 \mathrm{M} \mathrm{Hz})$ operating at the second harmonic $\left(\lambda_{p}=397.5 \mathrm{~nm}\right)$ with wavevector $-\mathbf{k}_{p}$, i.e. directed towards 
the left in Fig. 1. The emitted radiation $(\lambda=795 \mathrm{~nm})$ and the laser beam are then back-reflected by a spherical mirror $M$ with radius $R=15 \mathrm{~cm}$, highly reflecting both $\lambda$ and $\lambda_{p}$, placed at a distance $d=R$ from the NL crystal. A zero-order $\lambda / 4$ waveplate (wp), placed between $M$ and the BBO, performs the $|H H\rangle \rightarrow|V V\rangle$ transformation on the 2-photon state belonging to the left-cone while leaving in its original polarization state the pump beam $\left(\lambda_{p}=\lambda / 2\right)$ [15. The back-reflected laser beam excites an identical albeit distinct downconversion process with emission of a new radiation cone directed towards the right in Fig. 1 with axis $\mathbf{k}_{p}$. In this way, the state of the overall radiation, resulting from the two overlapping indistinguishable cones, is then expressed by the pure entangled state:

$$
|\Phi\rangle=\frac{1}{\sqrt{2}}\left(\left|H_{1}, H_{2}\right\rangle+e^{i \theta}\left|V_{1}, V_{2}\right\rangle\right)
$$

In the present source the particular configuration of single-arm interferometer allows to overcome many of the instability problems due to the typical phase fluctuations of a standard two arm interferometer. Phase stability arises from the fact that the superposition of SPDC emission cones takes place with phase $(0 \leq \phi \leq \pi)$ reliably controlled by micrometric displacement $(0 \leq \Delta d \leq 70 \mu m)$ of the spherical mirror $M$ along $\mathbf{k}_{p}[15]$.

A positive lens transforms the overall conical emission distribution into a cylindrical one whose transverse circular section identifies the so-called entanglement-ring (e-ring), with diameter $D=1.6 \mathrm{~cm}$.

The insertion of a zero-order $\lambda / 2 \mathrm{wp}$ in the signal or the idler arm (cfr. Fig. 2) makes possible to locally transform Eq.(3) into the state:

$$
|\Psi\rangle=\frac{1}{\sqrt{2}}\left(\left|H_{1}, V_{2}\right\rangle+e^{i \theta}\left|V_{1}, H_{2}\right\rangle\right)
$$

Then, by setting $\theta=0$ or $\theta=\pi$ the state can be locally transformed in any one of the four Bell states, Eqq. (11) and (2).

By this scheme it is possible to manipulate the superposition state by acting separately on one of the two emission cones. In particular, by a simple patchwork technique, pure states can be easily transformed into mixed states with various degree of mixedeness. Relevant classes of quantum states as the Werner States and the Maximally Entangled Mixed States (MEMS) have been created by this source [16, 17].

Besides polarization entanglement, in the present experiment, momentum (path) entanglement of two photons has been performed by selecting two pairs of symmetric points of the e-ring, $a_{1}-b_{2}$ and $a_{2}-b_{1}$ (Fig. 1: inset), 
following the idea proposed in Ref. [13]. The diameters connecting $a_{1}$ to $b_{2}$ and $a_{2}$ to $b_{1}$ intercross at the angle $\alpha$ (see Fig. 1). The unitary character of the SPDC transformation allows the biphoton state to keep the same phase of the pump beam, regardless the value of $\alpha$. Hence the following expression of the momentum entangled Bell states holds for either one of the two emission cones:

$$
\left|\psi^{ \pm}\right\rangle=\frac{1}{\sqrt{2}}\left(\left|a_{1}, b_{2}\right\rangle \pm\left|b_{1}, a_{2}\right\rangle\right) .
$$

In this experiment the phase is setted, $\phi=0, \phi=\pi$, by the insertion of two thin glass plates intercepting the pairs $a_{1}-b_{2}$ and $a_{2}-b_{1}$, and by a suitable tilting of one of the two plates (Fig. 1: inset). Several masks with different values of $\alpha$, each one with four holes of diameter $d=1.5 \mathrm{~mm}$, corresponding to the points $a_{1}, a_{2}, b_{1}, b_{2}$, have been tested..They are mounted on a rotation stage to optimize the quality of the momentum entanglement.

The SPDC outcoming radiation is divided along a vertical axis in two sets of modes, $a_{1}-b_{1}$ and $a_{2}-b_{2}$, by a prism-like two-mirror system and then reflected towards a multimode Hong-Ou-Mandel (HOM) interferometer [18] (Fig. 2). Such a measurement appears to be the most direct one to test the entanglement character of the state in the two degrees of freedom of polarization and momentum. In the inset of Fig. 2 it is shown how the modes $a_{1}$ and $b_{1}$ recombine with the corresponding modes $a_{2}$ and $b_{2}$ on the plane of a nonpolarizing $50-50$ beam splitter (BS). A trombone mirror assembly mounted on a step by step translation stage allows the fine adjustment of the path delay $\Delta x$ between the mode sets $a_{1}-b_{1}$ and $a_{2}-b_{2}$.

The outcoming signals belonging to the output BS modes, $a_{1}^{\prime}-b_{1}^{\prime}$ and $a_{2}^{\prime}-b_{2}^{\prime}$, are focused on the active surfaces of two independent avalanche single photon detectors, mod. SPCM-AQR14. Two equal interference filters, with bandwidth $\Delta \lambda=3 \mathrm{~nm}$, placed in front of the detectors, determine the coherence-time of the emitted photons: $\tau_{\text {coh }} \approx 400 \mathrm{f} \mathrm{sec}$.

\section{Experimental results:}

\section{1 a) Polarization entanglement}

We have characterized first the polarization entangled states $\left|\Psi^{ \pm}\right\rangle$generated by the source. For this measurements the four hole mask has been rotated

in order to get the SPDC radiation passing only through the symmetric holes $a_{1}$ and $b_{2}$ (or, equivalently, $a_{2}$ and $b_{1}$ ) alligned in the $H$ direction .When 
operating in the femtosecond regime the source is affected by a large temporal walk-off effect between the $H$ and $V$ polarization components. It comes out that, because of double passage through $\mathrm{BBO}$ and the $\lambda / 4 \mathrm{wp}$, the product state $\left|V_{1}, V_{2}\right\rangle$, corresponding to the SPDC radiation generated toward the left of Fig. 1, is advanced by $\sim 540 \mathrm{f}$ sec with respect the state $\left|H_{1}, H_{2}\right\rangle$, generated toward the right. As a consequence, due to the temporal width of the biphoton wavepacket, the two polarization components do not overlap. Temporal indistinguishability is recovered by means of a $18 \mathrm{~mm}$ quartz plate $(Q)$ intercepting the entire e-ring, aligned at the output of the source with the optic axis oriented along the $H$ direction (Fig. 2).

We have tested the polarization entanglement by means of two standard polarization analyzer settings (not shown in Fig. 2) in front of the detectors. Without the BS in Fig. 2, a polarization interference visibility of $\sim 90 \%$ has been measured for the Bell state $\left|\Phi^{-}\right\rangle[19]$.

By inserting the BS and removing the polarization analyzers, standard HOM interferometric tests have been performed for the Bell states $\left|\Psi^{ \pm}\right\rangle=$

$\frac{1}{\sqrt{2}}\left(\left|H_{1}, V_{2}\right\rangle \pm\left|V_{1}, H_{2}\right\rangle\right)$, corresponding to two different positions of mirror $M$ with respect the NL crystal. The experimental results given in Fig. 3a, clearly indicate the typical dip-peak interference due to the entangled nature of the state, corresponding to a visibility: $V=(0.87 \pm 0.01)$. The FWHM $(\simeq 60 \mu \mathrm{m})$ of the interference pattern is in agreement with the expected value for a filter bandwidth $\Delta \lambda=3 \mathrm{~nm}$. We have also tested the coincidence fringe visibility for a path length difference $\Delta x=0$, by varying the phase $\theta$ with the position of mirror $M$. The results given in Fig. 3b show the expected interference behaviour of the number of coincidences $N_{C}(|\Psi\rangle) \propto 1-\cos \theta$, with periodicity $\sim 70 \mu m[20$.

\section{2 b) Momentum entanglement}

The momentum entangled states $\left|\psi^{ \pm}\right\rangle$have been investigated with the four hole mask adjusted as shown in Fig. 1. The radiation belonging to either one of the emission cones of the source (cfr.Fig. 1) has been detected and measured. Different values of the angle $\alpha$, ranging from $10^{\circ}$ to $40^{\circ}$, have been tested. In all the cases the typical Hong-Ou-Mandel dip behaviour has been observed as a function of $\Delta x$ for the state $\left|\psi^{+}\right\rangle, \phi=0$ in Eq.(15).

All the possible falsification tests have been performed to prove the entanglement character of the state. In particular we have found that the dip disappears when either one of the mode pairs, $a_{1}-b_{2}$, or $a_{2}-b_{1}$ is covered. 
On the other hand no coincidence is observed when either the hole pairs of the rotating mask $a_{1}-a_{2}$ or $b_{1}-b_{2}$ are covered.

The main proof of the existance of momentum entanglement is given by the possibility of manipulating the phase of the state $|\psi\rangle$, viz. by tilting one of the glass plates of Fig. 1. Similarly to the case of polarization entanglement, Fig. 4a shows the triplet-singlet transition, $\phi=0 \rightarrow \phi=\pi$, obtained in the HOM interferometer with an interference visibility is $V=(0.82 \pm 0.01)$. The oscillation behaviour $N_{C}(|\psi\rangle) \propto 1-\cos \phi$, obtained for $\Delta x=0$, as a function of $\phi$ is given in Fig. $4 \mathrm{~b}[20$.

\section{3 c) Hyper-entanglement}

The hyper-entangled state realized in the present experiment is given by the factorization of the Bell states representing the entanglement in polarization and momentum, $|\Xi\rangle=\left|\Psi^{ \pm}\right\rangle \otimes\left|\psi^{ \pm}\right\rangle$. It can be optimized by the simultaneous, independent manipulation of the phases $\theta$ and $\phi$. We can express the state $|\Xi\rangle$ as

$$
|\Xi\rangle=\frac{1}{2}\left\{\left|H_{1}, V_{2}\right\rangle+e^{i \theta}\left|V_{1}, H_{2}\right\rangle\right\}\left\{\left|a_{1}, b_{2}\right\rangle+e^{i \phi}\left|b_{1}, a_{2}\right\rangle\right\}
$$

In terms of the creation operators of the electromagnetic field applied to the vacuum state, we can write

$$
|\Xi\rangle=\frac{1}{2}\left\{a_{1 H}^{\dagger} b_{2 V}^{\dagger}+e^{i \theta} a_{1 V}^{\dagger} b_{2 H}^{\dagger}+e^{i \phi} b_{1 H}^{\dagger} a_{2 V}^{\dagger}+e^{i(\theta+\phi)} b_{1 V}^{\dagger} a_{2 H}^{\dagger}\right\}|0\rangle,
$$

where $a_{j \sigma}^{\dagger}$ and $b_{j \sigma}^{\dagger}$ represent the operators associated to the modes $a_{j}$ and $b_{j}$, respectively, and to the polarization $\sigma(j=1,2$ and $\sigma=H, V)$. In a HOM interference experiment the operator relations existing between the input and output modes of the BS 21] allow to express the state $|\Xi\rangle$ as

$$
|\Xi\rangle=\frac{1}{4}\left\{\begin{array}{c}
{\left[\begin{array}{c}
a_{1 H}^{\dagger^{\prime}} b_{2 V}^{\dagger^{\prime}}\left(1-e^{i(\theta+\phi)}\right)-i a_{2 H}^{\dagger^{\prime}} b_{2 V}^{\dagger^{\prime}}\left(1+e^{i(\theta+\phi)}\right) \\
-i a_{1 H}^{\dagger^{\prime}} b_{1 V}^{\dagger^{\prime}}\left(1+e^{i(\theta+\phi)}\right)-a_{2 H}^{\dagger^{\prime}} b_{1 V}^{\dagger^{\prime}}\left(1-e^{i(\theta+\phi)}\right)
\end{array}\right]+} \\
+e^{i \theta}\left[\begin{array}{c}
a_{1 H}^{\dagger^{\prime}} b_{2 V}^{\dagger^{\prime}}\left(1-e^{i(\phi-\theta)}\right)-i a_{2 V}^{\dagger^{\prime}} b_{2 H}^{\dagger^{\prime}}\left(1+e^{i(\phi-\theta)}\right) \\
-i a_{1 V}^{\dagger^{\prime}} b_{1 H}^{\dagger^{\prime}}\left(1+e^{i(\phi-\theta)}\right)-a_{2 V}^{\dagger^{\prime}} b_{1 H}^{\dagger^{\prime}}\left(1-e^{i(\phi-\theta)}\right)
\end{array}\right]
\end{array}\right\}|0\rangle
$$

Hence the expected number of coincidences at the output of HOM is:

$$
N_{C}(|\Xi\rangle)=\frac{1}{2}(1-\cos \phi \cos \theta) \text {. }
$$


This behaviour can be easily understood by considering that, when $\theta=0$, $\phi=0$ or $\theta=\pi, \phi=\pi$, the symmetry of the state $|\Xi\rangle$ is bosonic, hence we expect the photons go together through the same arm of the BS. On the contrary, for $\theta=0, \phi=\pi$ or $\theta=\pi, \phi=0$, the state $|\Xi\rangle$ is characterized by a fermionic symmetry and the photons go separately into the two output arms of the BS.

The experimental results of Fig. 5 verify the bosonic-fermionic transition predicted for the state $|\Xi\rangle$ by the expression (7). In this case the phase $\phi$ has been varied by tilting one of the glass plates for two given positions of mirror $M$, corresponding to the phase values $\theta=0, \pi$.

An accurate experimental production of the hyper-entangled state has been found particularly severe, likely because of the general critical requirements needed for operating on the simultaneous conditions of entanglement in the two degrees of freedom of polarization and momentum. This is confirmed by the value of the oscillation visibility, $V \simeq 60 \%$ obtained in this experiment. We do believe that the experimental difficulties due to the need of a simultaneous superposition of the modes in two different region of BS and a possible unperfect modal structure of the pump beam may be also responsible of this problem.

\section{Conclusion}

The experimental realization of two photon states, simultaneously entangled in polarization and momentum, so-called hyper-entangled states, has been presented in this paper. These states have been realized by selecting two pairs of correlated $\mathbf{k}$-vectors within the degenerate emission cone of a high brilliance source of polarization entangled photons, recently developed by us. Hyper-entangled states are manipulated by varying independently the phases corresponding to the two entanglement degrees of freedom of the states. We have investigated the entanglement character of these states by multimode HOM interferometry. They can be symmetric or antisymmetric, depending on the combined values of the two phases. The additional degree of freedom given by the possibility of varying either one of the two phases, may represent a useful control parameter in quantum state engineering and Bell state measurements.

This work was supported by the FET European Network on Quantum Information and Communication (Contract IST-2000-29681: ATESIT), MIUR 
2002-Cofinanziamento and PRA-INFM 2002 (CLON).

\section{References}

[1] A.Ekert, R. Jozsa, R., 1996, Rev. Mod. Phys. 68, 733; E.Knill, R. Laflamme, G.J. Milburn, 2001, Nature 409, 46.

[2] C. H. Bennett, G. Brassard, C. Crepeau, R. Jozsa, A. Peres and W. K. Wootters, 1993, Phys. Rev. Lett. 70, 1895.

[3] A.Ekert, 1992, Nature 358, 14; N. Gisin, G. Ribordy, W. Tittel, and H. Zbinden, 2002, Rev. Mod. Phys. 74, 145.

[4] C. Bennett and S. J. Wiesner, 1992, Phys. Rev. Lett. 69, 2881; K. Mattle, H. Weinfurter, P. G. Kwiat and A. Zeilinger, 1996, Phys. Rev. Lett. 76, 4656 .

[5] D. Boschi, S. Branca, F. De Martini, L. Hardy and S. Popescu, 1998, Phys. Rev. Lett. 80, 1121; D. Bouwmeester J. M. Pan, K. Mattle, M. Eibl, H. Weinfurther and A. Zeilinger, 1997, Nature (London) 390, 575;

I. Marcikic et al., 2003, Nature (London) 421, 509.

[6] J. W. Pan, D. Bouwmeester, H. Weinfurter and A. Zeilinger, 1998, Phys. Rev. Lett. 80, 3891; T. Jennewein, G. Weihs, J. W. Pan and A. Zeilinger, 2002, Phys. Rev. Lett. 88, 017903; F. Sciarrino, E. Lombardi, G. Milani and F. De Martini, 2002, Physical Review A 66, 024309.

[7] D. Klyshko, Photons and Nonlinear Optics (Gordon and Breach, New York, 1988).

[8] S. Ghosh, G. Kar, A. Roy, A. Sen (De) and U. Sen, 2001, Phys. Rev. Lett. 87, 277902; J. Calsamiglia, N. Lukenhaus, 1999, Appl. Phys. B: Lasers Opt., 72, 67.

[9] P. G. Kwiat and H. Weinfurther, 1998, Phys. Rev. A, 58, R2623.

[10] P. G. Kwiat, K. Mattle, H. Weinfurter and A. Zeilinger 1995, Phys. Rev. Lett. 75, 4337.

[11] Z.B Chen, J.W. Pan, Y.D. Zhang, C. Brukner, A. Zeilinger, 2003, Phys. Rev. Lett., 90, 160408. 
[12] P. G. Kwiat, E. Waks, A. G. White, I. Appelbaum and P. H. Eberhard, 1999, Phys. Rev. A, 60, R773.

[13] S. P. Walborn, W. S. Padùa and C. H. Monken, 2003, Phys. Rev. A 68, 042313.

[14] G. Giorgi, G. Di Nepi, P. Mataloni and F. De Martini, 2003, Laser Physics, 13, 350 .

[15] C. Cinelli, G. Di Nepi, M. Barbieri, F. De Martini and P. Mataloni, "A parametric source of two-photon states with tunable degree of entanglement and mixedeness: experimental preparation of Werner states and Maximally Entangled Mixed States", quant-ph/0307204, submitted to Phys. Rev. A.

[16] M. Barbieri, F. De Martini, G. Di Nepi, P. Mataloni, "Generation and characterization of Werner states and maximally entangled mixed states by a universal source of entanglement", 2004, Phys. Rev. Lett.,92, 177901.

[17] M.Barbieri, F.De Martini, G.Di Nepi, P.Mataloni, G.M.D'Ariano and C.Macchiavello, 2003, Phys.Rev.Lett 91, 227901.

[18] C. K. Hong, Z. Y. Ou and L. Mandel, 1987, Phys. Rev. Lett. 59, 2044; S.P. Walborn, A. N. de Oliveira, S. Pàdua, C. H. Monken, 2003, Phys. Rev. Lett. 90, 143601.

[19] Z. Y. Ou and L. Mandel, 1988, Phys. Rev. Lett. 61, 50.

[20] D. Bouwmeester, A. Ekert, A. Zeilinger, The Physics of Quantum Information (Springer-Verlag, Berlin 2000).

[21] R. Loudon, The Quantum Theory of Light Ch.5 (Clarendon Press, Oxford 2000).

\section{FIGURE CAPTIONS}

Fig. 1 - Generation of hyper-entangled two photon states: experimental setup. Displacement of mirror $M$ allows to change the phase $\theta$. Inset: four hole mask and glass plate system for phase $\phi$ adjustment.. 
Fig. 2 - Multimode HOM interferometer. After walk-off compensation $(Q)$, the mode sets $a_{1}-b_{1}$ and $a_{2}-b_{2}$, travelling along the two arms of the interferometer, are recombined onto a nonpolarizing symmetric BS by translation $\Delta x$. Inset: spatial coupling of the input modes $a_{1}-b_{1}$, $a_{2}-b_{2}$ on the BS. The BS output modes, $a_{1}^{\prime}-b_{1}^{\prime}, a_{2}^{\prime}-b_{2}^{\prime}$ are also shown.

Fig. 3 - a) Coincidence rate depending on the path length difference $\Delta x$ for the polarization entangled states $\left|\Psi^{+}\right\rangle(\theta=0)$ and $\left|\Psi^{-}\right\rangle(\theta=\pi)$. b) Coincidence rate measured as a function of the mirror $M$ position $(\Delta x=0)$.

Fig. 4 - a) Coincidence rate depending on the path length difference $\Delta x$ for the momentum entangled states $\left|\psi^{+}\right\rangle(\phi=0)$ and $\left|\psi^{-}\right\rangle(\phi=\pi)$. b) Coincidence rate measured as a function of phase $\phi$, expressed in units of $\pi .(\Delta x=0)$.

Fig. 5 - Coincidence rate vs. $\phi$ (in units of $\pi$ ) for the hyper-entangled state $|\Xi\rangle(\Delta x=0)$. Continuous line: $\theta=0$, dashed line: $\theta=\pi$. 


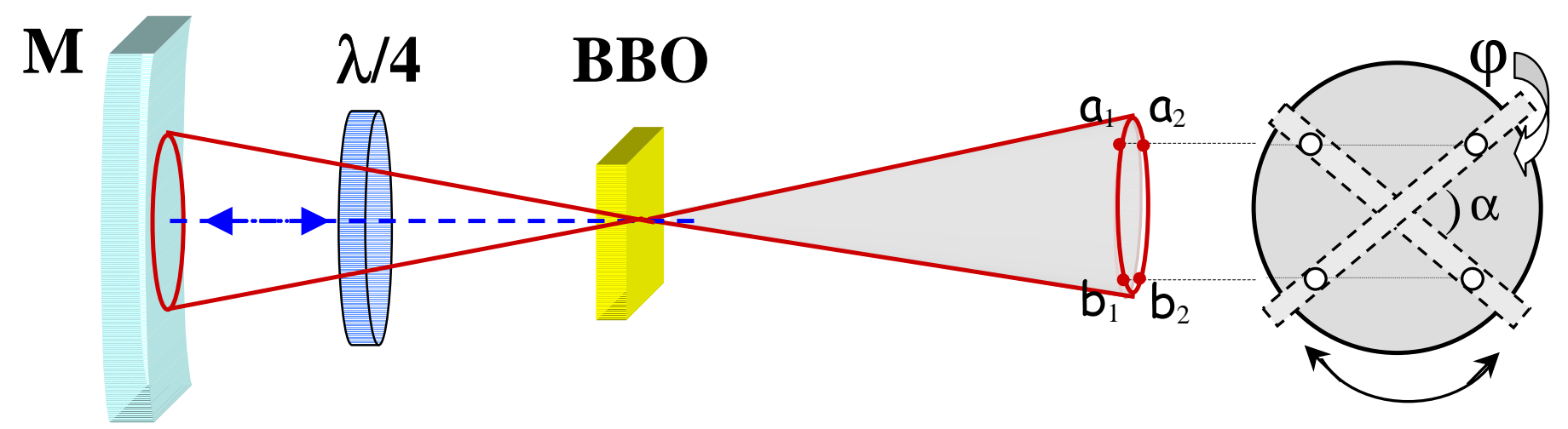




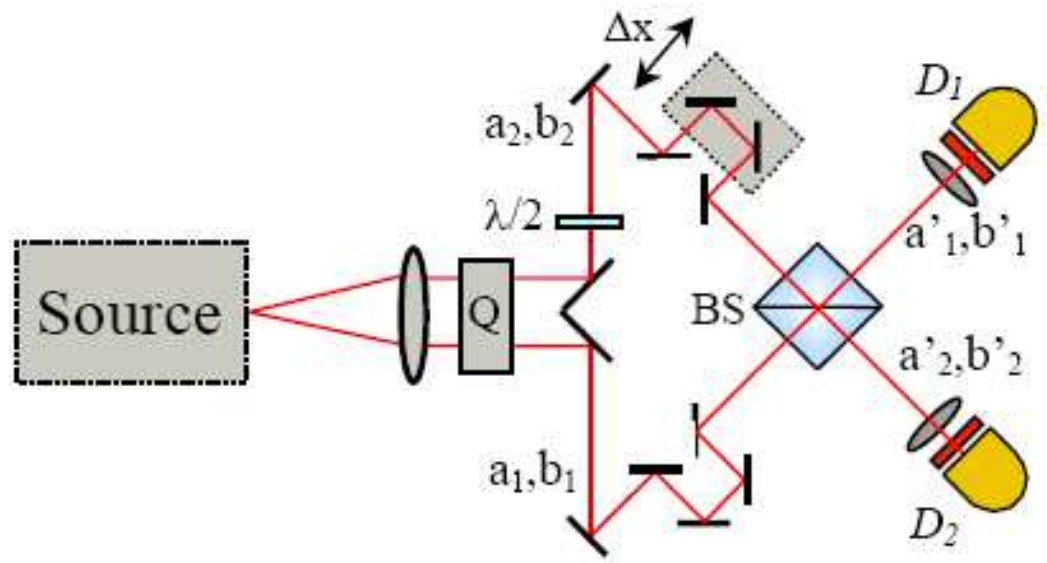

a)

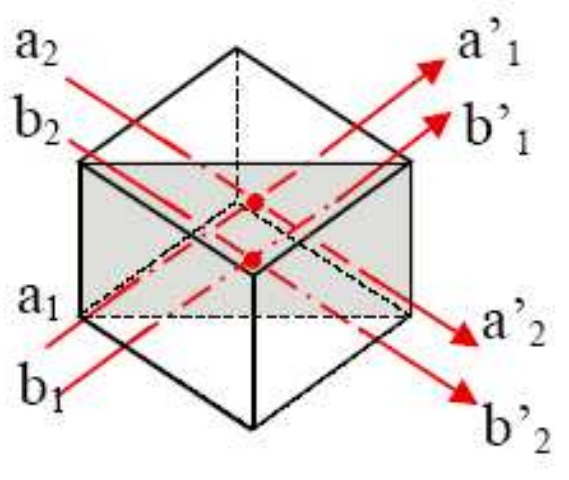

b) 

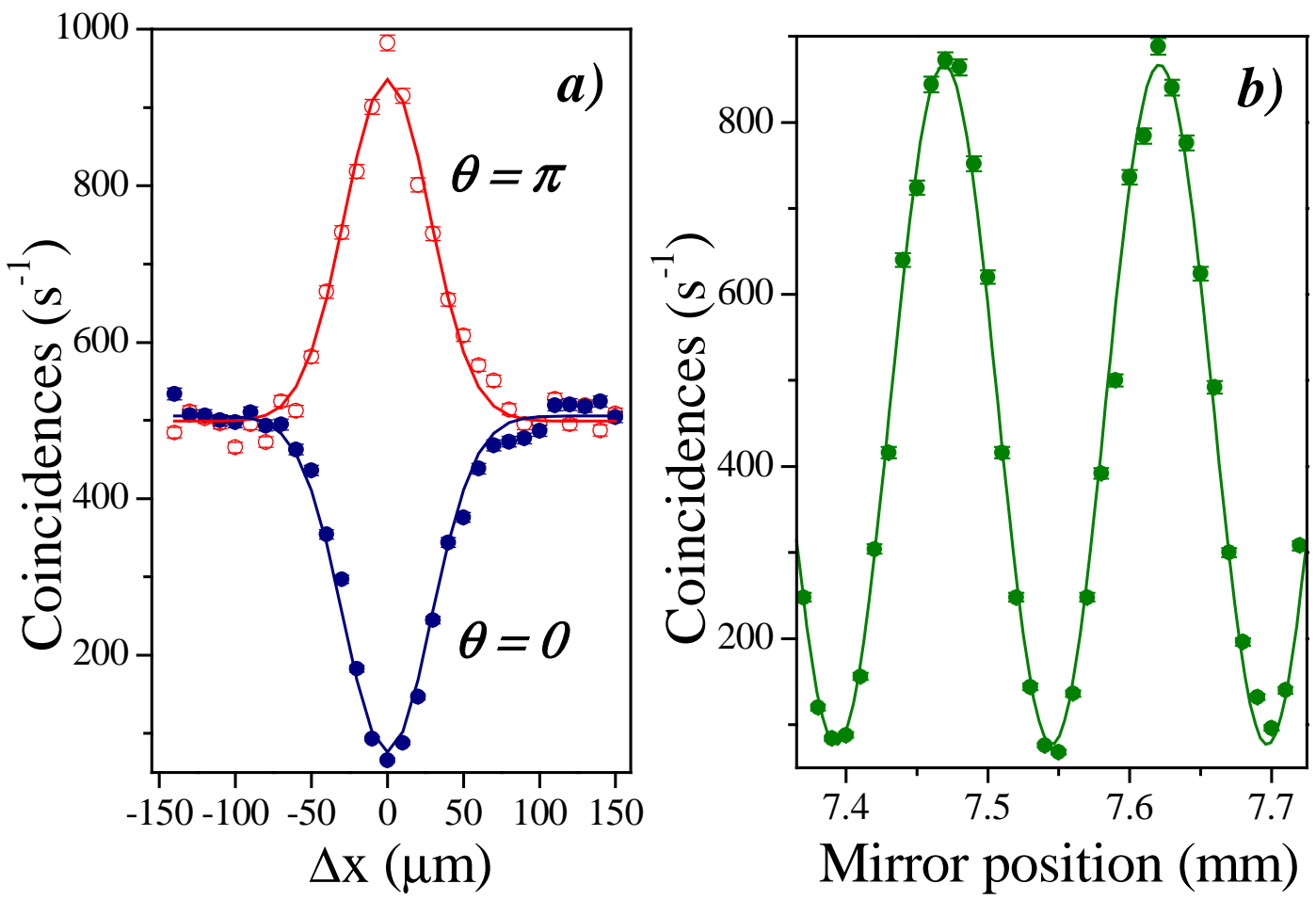

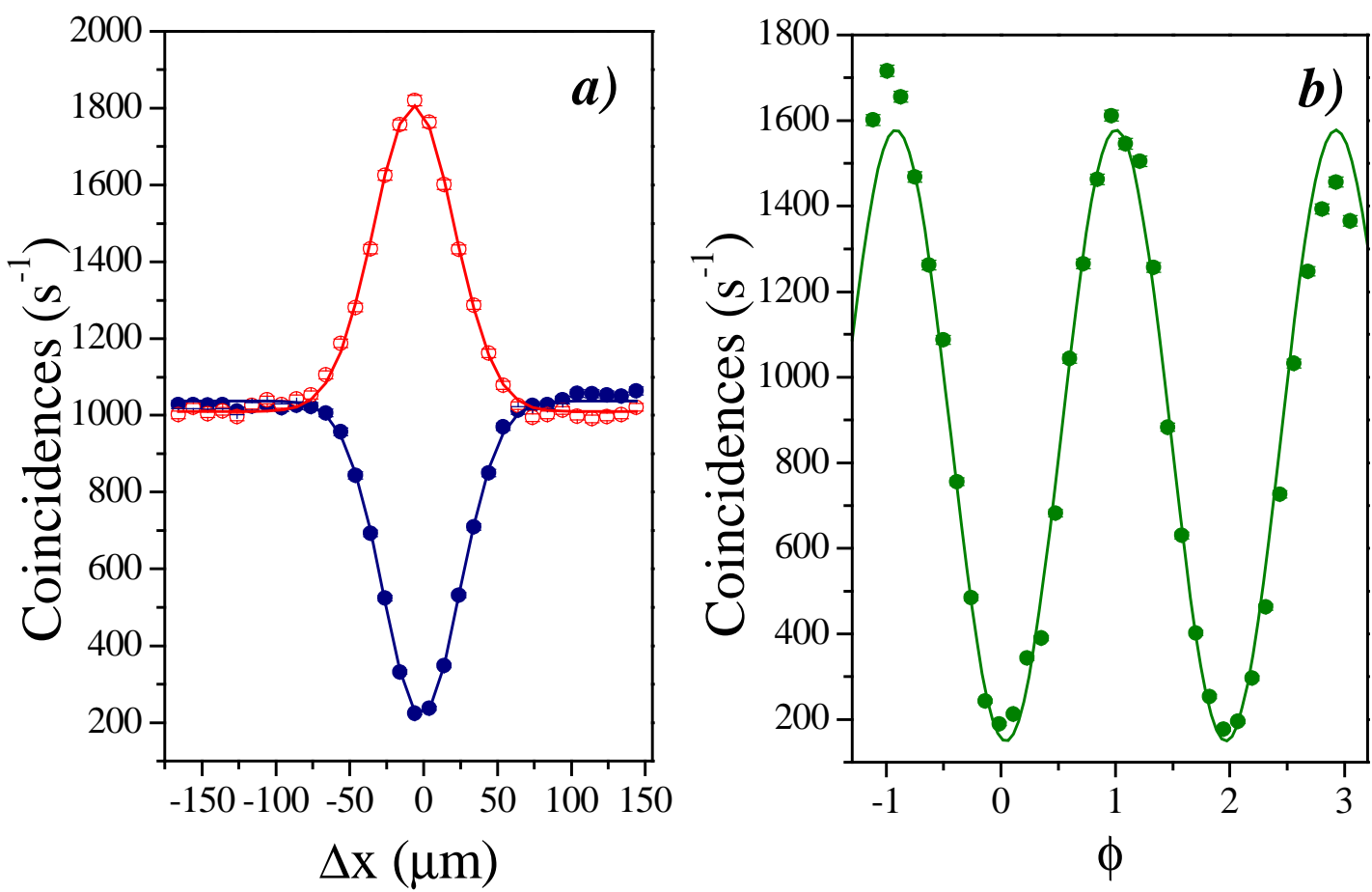


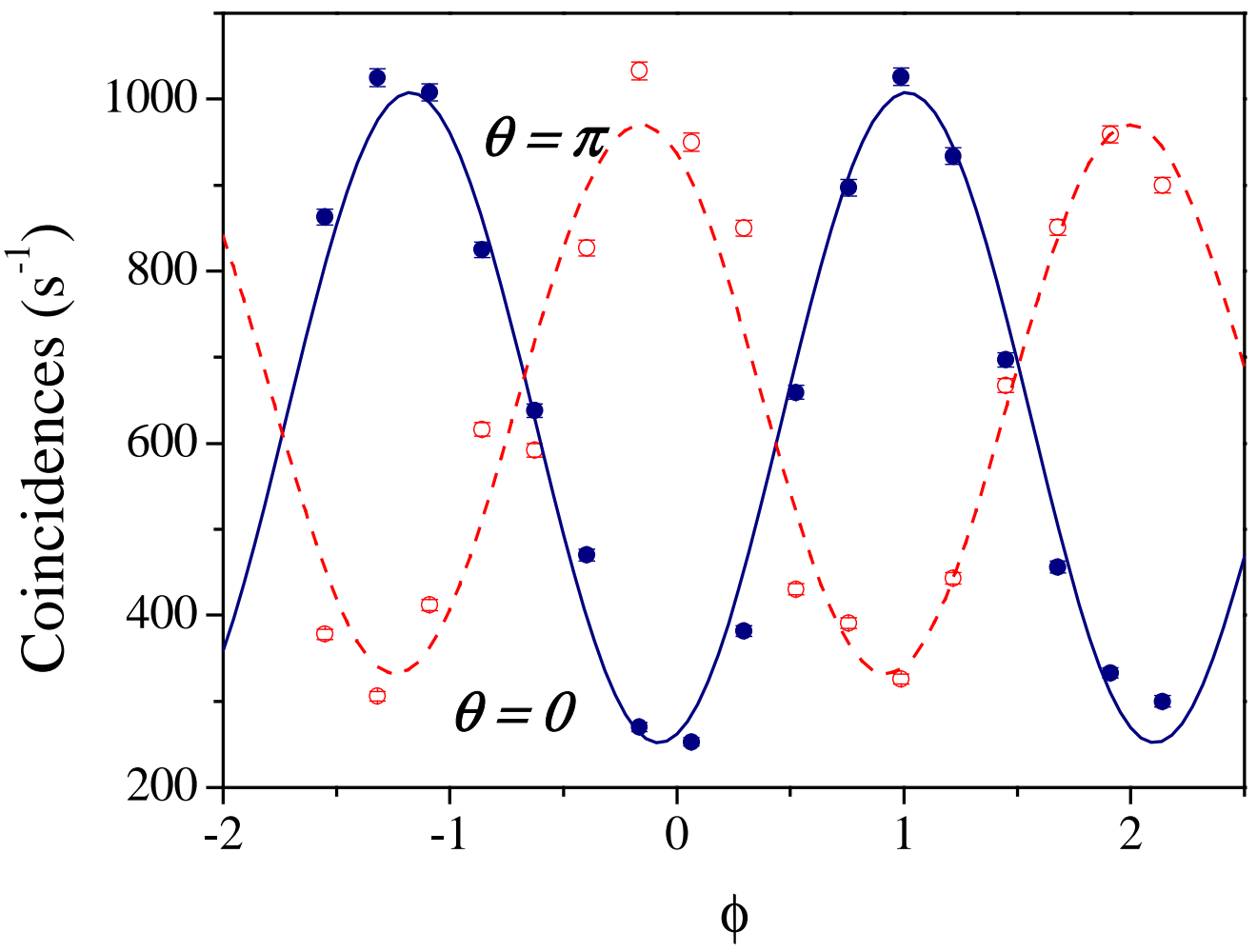

